\title{
ADIABATIC COMPRESSION OF PLASMA WITH PURELY TOROIDAL ROTATION
}

\author{
L. J. PAN \\ Institute of Mechanics, Chinese Academy of Sciences, Beijing, People's Republic of China and \\ Department of Physics, Imperial College, London SW7, U.K. \\ and \\ D. Fu \\ Institute of Mechanics, Chinese Academy of Sciences, Beijing, People's Republic of China \\ (Received 6 April 1987 ; and in revised form 12 January 1989)
}

\begin{abstract}
A variational method is developed to find approximate solutions to the generalized GradShafranov equations for an adiabatic compression of the plasma with toroidal rotation, via the expansion in Fourier series in poloidal angle of the flux surface coordinates. The numerical results, which are carried out by the present method and by the usual two-dimensional method for a static equilibrium state, agree well.
\end{abstract}

\section{INTRODUCTION}

THE AIM OF this work is to develop a variational method for seeking the approximate solutions to the generalized Grad-Shafranov equation for the adiabatic compression of plasma with purely toroidal rotation. We have treated the adiabatic compression of a plasma with both poloidal and toroidal rotation (PAN, 1989) under an assumption that the Mach number is small, which is removed in the present work. HAMEIRI (1983) has treated this problem by an extension of GRAD et al.'s (1975) "alternatingdimensions method" (ADM) via a variational principle based on a few constants of motion. For the evolving flux geometry, such a technique consisting of iterations between a partial differential equation and an ordinary equation via the variational step, significantly increases the computer time and storage requirements of a transport code.

We find that the variational method developed by LAO et al. (1981) for finding approximate solutions to the Grad-Shafranov equation for plasma without flow can be extended to our case.

\section{THE SYSTEM OF EQUATIONS}

The first assumption made in this paper is that the compression takes place on a time scale much slower than typical Alfvén wave transit time across the major dimensions of the device. The waves cause fast equilibrium; the plasma, therefore, can be viewed as creeping from one equilibrium state to the next. The second assumption is that the plasma is governed by an axisymmetric ideal MHD equation, although the final plasma state may be affected by transport processes. We assume that during compression the poloidal velocity is damped out by parallel viscous stress. The third assumption is that the temperature on each flux surface is constant due to the very 
TABLE 1.-Notations

\begin{tabular}{rlrl}
\hline$\psi_{1}$ & $=\psi / \psi_{\mathrm{m}}$ & $M$ & $=\sqrt{2 \alpha / \gamma R}, \quad \gamma=\frac{5}{3}$ \\
$\psi_{\mathrm{b}}$ & $=\psi_{\mathrm{m}} / \bar{B}_{\mathrm{p}} \cdot R_{0 a} a$ & $\alpha_{T}$ & $=\frac{1}{2} \Omega_{0}^{2} a^{2}$ \\
$P_{1}$ & $=P / P_{\mathrm{m}}$ & $\varepsilon$ & $=a / R_{0 a}$ \\
$P_{\mathrm{b}}$ & $=P_{\mathrm{m}} /\left(\bar{B}_{\mathrm{p}}^{2} / 2\right)$ & $\delta_{\mathrm{T}}$ & $=R_{2 a} / a$ \\
$I_{1}$ & $=I / \bar{B}_{\mathrm{p}} R_{0 a}=\sqrt{B_{\mathrm{t}} \psi_{1}^{2}+I_{10}^{2}} \cdot$ & $\bar{R}$ & $=R / a$ \\
$\beta_{\mathrm{p}}$ & $=\int P \mathrm{~d} v /\left(v \bar{B}_{\mathrm{p}}^{2} / 2\right)$ & $\bar{Z}$ & $=Z / a$ \\
$\beta_{\mathrm{t}}$ & $=\int P \mathrm{~d} v /\left(v \bar{B}_{\mathrm{t}}^{2} / 2\right)$ & $X$ & $=\eta / a$ \\
$q$ & $=\frac{I}{4 \pi^{2}}\left\langle\frac{1}{R^{2}}\right\rangle \frac{\mathrm{d} v}{d \psi}$ & $\bar{R}_{0}$ & $=R_{0} / a$ \\
$a$ & $=\frac{1}{2} \Omega_{0}^{2} R^{2}$ & $\bar{R}_{2}$ & $=R_{2} / a$
\end{tabular}

The subscript $m$ denotes the quantity at the magnetic axis, and $\eta=a$ is the plasma boundary.

high heat conductivity along a magnetic field. It means that the plasma we treated is slightly non-ideal, but more realistic. Under these assumptions, all the related equations given by HAMEIRI (1983) are written in the following. The notations used are listed in Table 1.

\subsection{The generalized Grad-Shafranov equation}

$$
\nabla \cdot\left\{\frac{1}{R^{2}} \nabla \psi\right\}+\left(R^{2} \Omega_{0} \dot{\Omega}_{0}+\dot{H}_{0}\right) P+\frac{1}{R^{2}} I \dot{I}=0
$$

where

$$
\begin{gathered}
\text { ". }=\frac{\mathrm{d}}{\mathrm{d} \psi} \\
P=\exp \left(H_{0}+\frac{1}{2} R^{2} \Omega_{0}^{2}\right) \\
\Omega_{0}^{2}=\Omega^{2} / T, \quad H_{0}=H / T+\ln T \\
U_{\phi}=R \Omega \\
B_{\phi}=I / R
\end{gathered}
$$

and 


$$
P=\rho T=S \rho^{\gamma}, \quad S=\mathrm{e}^{s}
$$

The cylindrical coordinates $(R, \phi, Z)$ are adopted. $2 \pi \psi$ is defined as the magnetic flux enclosed between the flux surface and the axis of symmetry. $T(\psi), I(\psi), H(\psi)$ and $\Omega(\psi)$ are four arbitrary functions in $(1) ; u, T, P, \rho$ and $s$ denote the plasma toroidal velocity, temperature, pressure, density and specific entropy, respectively. $I(\psi)$ in $(5)$ is the poloidal current enclosed between the axis of symmetry and the magnetic surface and determines the toroidal magnetic field. Relation (6) is the equation of state with $\gamma$ the adiabatic constant. The function $\psi(R, Z)$ is determined from (1). $\Omega(\psi)$ is the frequency of rotation.

\subsection{Conservation-law constraints}

Mass conservation

$$
\langle\rho\rangle=m \psi^{\prime}
$$

angular momentum

$$
\left\langle R \rho U_{\phi}\right\rangle=l(\psi) \psi^{\prime}
$$

toroidal magnetic field

$$
\left\langle B_{\phi} / R\right\rangle=f(\psi) \psi^{\prime}
$$

total entropy inside each surface

$$
\langle\rho S\rangle=\sigma(\psi) \psi^{\prime}
$$

where

$$
\frac{\mathrm{d}}{\mathrm{d} v} \int_{\psi} f \mathrm{~d} v=\oint f \mathrm{~d} \Sigma /|\nabla V|=\langle f\rangle,()^{\prime}=\frac{\mathrm{d}}{\mathrm{d} v}
$$

where $m, l, f$ and $\sigma$ are unchanged during compression, and $\Sigma, V$ and $\mathrm{d} v$ represent the area of a flux surface, the volume inside a closed flux surface and the volume element, respectively. Equations (7)-(10) indicate that, within each moving flux tube, the plasma mass, angular momentum, toroidal magnetic flux and the total entropy remain constant during compression. Equation (10) implies that no heat flows across flux surfaces.

\section{ANALYTIC FORMULATION}

\subsection{Variational principle}

Consider the functional $Q$ defined as the volume integral

$$
Q=\int_{V} L\left(\psi, \psi_{R}, \psi_{Z}, R\right) R \mathrm{~d} R \mathrm{~d} Z
$$

Here the Lagrangian associated with equation (1) is given by

$$
L=\frac{1}{2 R^{2}}(\nabla \psi)^{2}-\frac{I^{2}}{2 R^{2}}-\exp \left(\frac{1}{2} R^{2} \Omega_{0}^{2}+H_{0}\right)
$$


which can be found even by inspection, and the volume integral is over the entire volume of plasma, $V$. The variation of $Q$ with respect to $\psi$ yields the Euler equation

$$
\frac{\partial L}{\partial \psi}-\frac{\partial}{\partial R} \frac{\partial L}{\partial \psi_{R}}-\frac{\partial}{\partial} \frac{\partial L}{\partial \psi_{Z}}=\nabla \cdot\left(\frac{1}{R^{2}} \nabla \psi\right)+P\left(R^{2} \Omega_{0} \dot{\Omega}_{0}+\dot{H}_{0}\right)+\frac{1}{R^{2}} I \dot{I} .
$$

It implies that the assumed Lagrangian in (13) is correct and that $Q$ is stationary for $\psi$ satisfying the generalized Grad-Shafranov equation (1). The key idea of the variational method is to convert a two-dimensional problem to a one-dimensional problem by the choice of a convenient form of solutions, say a Fourier series, of which the amplitudes are determined in such a way that $Q$ is stationary. Its advantage will come to light later.

\subsection{Transformation of coordinates}

Consider the transformation of the cylindrical coordinates $(R, \phi, Z)$ to coordinates $(\eta, \theta, \Phi)$ with the relations

$$
R=R(\eta, \theta), \quad Z=Z(\eta, \theta),
$$

then it follows that the representation of $Q$ in new coordinates is

$$
\left.Q=\int_{0}^{a} \int_{0}^{2 \pi} L^{*}\left(R, R_{\eta}, R_{\theta}, Z_{\eta}, Z_{\theta}, \psi, \psi_{\eta}\right) \mathrm{d}_{\eta}\right) \mathrm{d} \eta \mathrm{d} \theta
$$

with the transformed Lagrangian

$$
\begin{aligned}
L^{*} & =\left(R_{\theta}^{2}+Z_{\theta}^{2}\right) \frac{\psi^{\prime 2}}{2 R^{\tau}}-R^{\tau}\left[\frac{I^{2}}{2 R^{2}}+\exp \left(\frac{1}{2} R^{2} \Omega_{0}^{2}+H_{0}\right)\right] \\
\tau & =R_{\theta} Z_{\eta}-R_{\eta} Z_{0}
\end{aligned}
$$

where $\eta$ is a function of $\psi, \phi$ is the ignorable toroidal angle and $\theta$ is the poloidal angle increasing $2 \pi$ the short way around the torus; $\eta=a$ denotes the outermost flux surface and the subscripts $\theta$ and $\eta$ denote the differentiation with respect to these variables.

The representation (16) consists of two dependent variables $R$ and $Z$ ( $\Phi$ plays the role of a coordinate). Since the stationary value of $Q$ is independent of any transformation, one can see that the variation of $Q$ with respect to $R$ and $Z$ subject to the boundary constraints $\delta R=\delta Z=0$ should reproduce the inverse generalized Grad-Shafranov equation. This has been proved by LAO et al. (1981) For clarity, the proof is briefly given in the following.

The variation of $Q$ with respect to $R$ and $Z$ under the fixed boundary constraints $\delta R=0$ and $\delta Z=0$ yield the Euler equations, respectively,

$$
-\frac{\partial L^{*}}{\partial R}+\frac{\partial}{\partial \eta} \frac{\partial L^{*}}{\partial R_{\eta}}+\frac{\partial}{\partial \theta} \frac{\partial L^{*}}{\partial R_{\theta}}=\psi^{\prime} Z_{\theta} \hat{G}=0
$$




$$
\frac{\partial}{\partial \eta} \frac{\partial L^{*}}{\partial Z_{\eta}}+\frac{\partial}{\partial \theta} \frac{\partial L^{*}}{\partial Z_{\theta}}=-\psi^{\prime} R_{\theta} \hat{G}=0
$$

with

$$
\begin{aligned}
\hat{G}=\frac{1}{\tau}\left[\frac{\partial}{\partial \eta}\left\{\frac{\partial}{\partial \eta}\left(\frac{\psi^{\prime}\left(R_{\theta}^{2}+Z_{\theta}^{2}\right)}{\tau R}\right)\right\}\right. & -\frac{\partial}{\partial \theta}\left\{\frac{\psi^{\prime}}{R \tau}\left(R_{\eta} Z_{\theta}+Z_{\eta} R_{\theta}\right)\right\} \\
& \left.+R\left\{\left(R^{2} \Omega_{0} \dot{\Omega}_{0}+H_{0}\right) \exp \left(\frac{1}{2} \Omega_{0}^{2} R^{2}+H_{0}\right)+I \dot{I} / R^{2}\right\}\right]
\end{aligned}
$$

One of the advantages for the use of the transformation (15) is that a free boundary problem for determining $\psi$ by (1) becomes a fixed boundary problem of solving the Euler equations (19) and (20) in coordinates $(\eta, \theta)$.

The proof indicates that the solution to an Euler equation is also a solution to the associated equation (1), therefore the variational method provides a new way to treat our complicated problem.

\section{APPROXIMATE SOLUTIONS IN TRUNCATED FOURIER SERIES}

\subsection{Convenient truncated Fourier series}

The specific one-to-one transformation in Fourier series developed by LAO et al. (1981)

$$
\begin{aligned}
& R(\eta, \theta)=R_{0}(\eta)-\eta \cos \theta+\sum_{n=2}^{n_{T}} R_{n}(\eta) \cos n \theta \\
& Z(\eta, \theta)=E(\eta)\left(\eta \sin \theta+\sum_{n=2}^{n_{T}} R_{n}(\eta) \sin n \theta\right)
\end{aligned}
$$

is adopted, where $\eta(\psi)$ is the flux label, the amplitudes $R_{0}(\eta),-\eta$ and $E$ describe the shift, the minor radius and the ellipticity of the flux surface, respectively, whereas the amplitudes $R_{2}(\eta)$ and $E R_{2}$ describe the triangularity of a flux surface.

One of the reasons for the use of the truncated Fourier series is that the main features of the magnetic field can be approximately described by a limited number of leading terms of the series.

\subsection{Euler equations for the Fourier coefficients}

It has been shown that the solutions to the Euler equation (14) or the transformed Euler equations (19) and (20) are the solutions to the generalized Grad-Shafranov equation (1). If a finite number of leading terms in the Fourier series are used to approximately describe the topology of the magnetic field, then the best choice of the Fourier amplitudes involved in the chosen terms can be obtained in such a way that $Q$ is stationary. 
The variation of $Q$ with respect to $R_{n}(\eta)$ and $E(\eta)$ generates a new set of Euler equations

$$
\begin{aligned}
& \left\langle\left\langle\frac{\partial L^{*}}{\partial R_{n}}-\frac{\partial}{\partial \eta} \frac{\partial L^{*}}{\partial R_{n}^{\prime}}\right\rangle\right\rangle=\left\langle\left\langle M_{n} \hat{G}\right\rangle\right\rangle=0 ; n=0,1, \ldots \\
& \left\langle\left\langle\frac{\partial L^{*}}{\partial E}-\frac{\partial}{\partial \eta} \frac{\partial L^{*}}{\partial E^{\prime}}\right\rangle\right\rangle=\left\langle\left\langle M_{E} \hat{G}\right\rangle\right\rangle=0 ; n=1,2, \ldots
\end{aligned}
$$

where the prime denotes differentiation with respect to $\eta$, and $\langle\langle\rangle\rangle$ is the poloidal angle averaging operator

$$
\langle\langle f\rangle\rangle=\int_{0}^{2 \pi} f \mathrm{~d} \theta / 2 \pi
$$

with

$$
\begin{gathered}
M_{0}=-Z_{\theta} \\
M_{1}=E R_{\theta} \sin \theta+Z_{\theta} \cos \theta \\
M_{n}=E R_{\theta} \sin n \theta-Z_{\theta} \cos n \theta \\
M_{E}=\sum_{n=1}^{n_{T}} R_{\theta} \sin n \theta .
\end{gathered}
$$

The above Euler equations (26)-(30) comprise a system of coupled, second order, nonlinear ordinary differential equations in variable $\eta$ for $R_{n}$ and $E$. Once the free functions $I, \Omega_{0}$ and $H_{0}$ involved in $G$ are given, all the Fourier amplitudes are then determined by the set of ordinary differential equations (24)-(30) with boundary conditions given in (42)-(47).

\subsection{Determination of $\mathrm{I}, \mathrm{H}_{0}, \Omega_{0}, \mathrm{~T}$ and $\rho$}

The five functions $I, H_{0}, \Omega_{0}, T$ and $\rho$ may be determined by the four constraints (7) $-(10)$ and the Bernoulli equation (2). The convenient form for $\rho$ satisfying (7) is

$$
\rho=m \psi^{\prime} \zeta, \quad \text { with }\langle\zeta\rangle=1
$$

where $\zeta$ may be viewed as the spatial dependence of $\rho$. Substituting (31) and (4) in the angular momentum constraint (8), one obtains

$$
\left\langle R^{2} \zeta\right\rangle \Omega=l / m
$$

By using state equation (6), (2) can be written as 


$$
\rho=\mathrm{e}^{\left(H_{0}+R^{2} \Omega_{0}^{2} / 2\right)} / T
$$

and taking a surface average we have

$$
T=\mathrm{e}^{H_{0}}\left\langle\mathrm{e}^{R^{2} \Omega_{0}^{2} / 2}\right\rangle / m \psi^{\prime}
$$

Thus (34), (33) and (31) give

$$
\zeta=\mathrm{e}^{R^{2} \Omega_{0}^{2} / 2} /\left\langle\mathrm{e}^{R^{2} \Omega_{0}^{2} / 2}\right\rangle .
$$

From (35), (32) and (34) we obtain

$$
\left.\mathrm{e}^{H_{0}}=l^{2} \psi^{\prime}\left\langle\mathrm{e}^{R^{2} \Omega_{0}^{2} / 2}\right\rangle /\left(m<R^{2} \mathrm{e}^{R^{2} \Omega_{0}^{2} / 2}\right\rangle^{2} \Omega_{0}^{2}\right) .
$$

Substitution of (6) and (31) in (10) and use of (35) lead to another relation

$$
H_{0}=\ln \left(\frac{m \psi^{\prime}}{\left\langle\mathrm{e}^{R^{2} \Omega_{0}^{2} / 2}\right\rangle}\right)^{\gamma}+\frac{\sigma}{m}+\frac{\gamma-1}{2} \Omega_{0}^{2}\left\langle R^{2} \mathrm{e}^{R^{2} \Omega_{0}^{2} / 2}\right\rangle\left\langle\mathrm{e}^{R^{2} \Omega_{0}^{2} / 2}\right\rangle
$$

Finally, elimination of $H_{0}$ from (36) and (37) results in

$$
\frac{l^{2} \psi^{(1-\gamma)}}{m^{(1+\gamma)}}\left\langle\mathrm{e}^{R^{2} \Omega_{0}^{2} / 2}\right\rangle^{\gamma+1}=\Omega_{0}^{2}\left\langle R^{2} \mathrm{e}^{R^{2} \Omega_{0}^{2} / 2}\right\rangle^{2} \exp \left[\frac{\frac{\sigma}{m}+\frac{\gamma-1}{2} \Omega_{0}^{2}\left\langle R^{2} \mathrm{e}^{R^{2} \Omega_{0}^{2} / 2}\right\rangle}{\left\langle\mathrm{e}^{R^{2} \Omega_{0}^{2} / 2}\right\rangle}\right] .
$$

Substitution of (5) in the toroidal magnetic flux constraint (9) yields the free function

$$
I=f \psi^{\prime} /\left\langle\frac{1}{R^{2}}\right\rangle
$$

Since on each surface all the unchanged constraint quantities $l, m$ and $\sigma$ in (37) and (38) are known, $H_{0}$ and $\Omega_{0}$ can be determined numerically by (37) and (38) with the given $R_{n}$.

With the three algebraic relations (37), (38) and (39), the set of Euler equations (24) and (25) can be solved for $R$ and $E$ through the boundary conditions prescribed by the compression method.

For small Mach number $R \Omega_{0},(37)$ and (38) give the approximate explicit expressions for $\Omega_{0}$ and $H_{0}$, respectively, by neglecting the order higher than $R_{0}^{2} \Omega_{0}^{2}$ as shown below :

$$
\Omega_{0}^{2}=\frac{l^{2}}{\mathrm{e}^{\sigma / m}\left\langle R^{2}\right\rangle^{2} m^{\gamma+1} \psi^{\prime(\gamma-1)}-\frac{(\gamma+1)}{2}\left\langle R^{2}\right\rangle l^{2}}
$$




$$
H_{0}=\ln \left(m \psi^{\prime}\right)^{\gamma}+\frac{\sigma}{m}-\frac{1}{2}\left\langle R^{2}\right\rangle \Omega_{0}^{2}
$$

Replacing the three free functions $I, H_{0}$ and $\Omega_{0}$ in $\hat{G}$ by (39), (40) and (41), respectively, we obtain new expressions for the Euler equations (24) and (25). Therefore the resulting set of nonlinear, second order, coupled ordinary differential equations can uniquely determine the required amplitudes $R_{n}$ and $E$. Consequently $\psi$ is determined under the prescribed boundary condition.

To conclude, in contrast with HAMEIRI's generalized ADM via a variational principle, the present variational method is a computationally fast, yet sufficiently accurate method for determining the evolving flux surface geometry.

\subsection{Boundary conditions}

\section{NUMERICAL SOLUTIONS}

Equations (22) and (23) are second order ordinary differential equations. The shape of the outermost flux surface provides one of the two sets of the boundary conditions:

when

$$
\begin{gathered}
\eta=a, \quad \psi(a)=0, \quad E(a)=E_{a} \\
R_{n}(a)=R_{n a}, \quad n=0,2,3 \ldots
\end{gathered}
$$

At the magnetic axis $\eta=0$, the amplitudes and derivatives of $R_{n}, R_{n}^{\prime}$ vanish, therefore $\eta=0$ is a critical point of the differential equations. LAO et al. (1981) give the forms of the solutions in the vicinity of $\eta=0$.

$$
\begin{gathered}
\psi=\psi_{\mathrm{m}}-\frac{E_{\mathrm{m}}^{2}}{2\left(1+E_{\mathrm{m}}^{2}\right)} \cdot\left(R_{\mathrm{m}}^{2} \dot{P}(0)+I(0) \dot{I}(0)\right) \eta^{2} \\
R_{0}=R_{\mathrm{m}}+\lambda_{1} \eta^{2} \\
R_{n}=R_{n 0} \eta^{n} \quad n=2, \ldots, \\
E=E_{\mathrm{m}}+\lambda_{2} \eta^{2}
\end{gathered}
$$

which may be viewed as another set of boundary conditions at small $\eta$, and $\lambda_{1}$ and $\lambda_{2}$ are arbitrary, small constants.

\subsection{Numerical method}

The numerical method is similar to the one in LAO et al. (1981). By substituting (44)-(47), and (40) and (41) into (24) and (25) one can determine the solutions by using a shooting technique and the Runge-Kutta method through the relations (44)(47).

Calculations have been carried out for static equilibrium states of plasmas in 


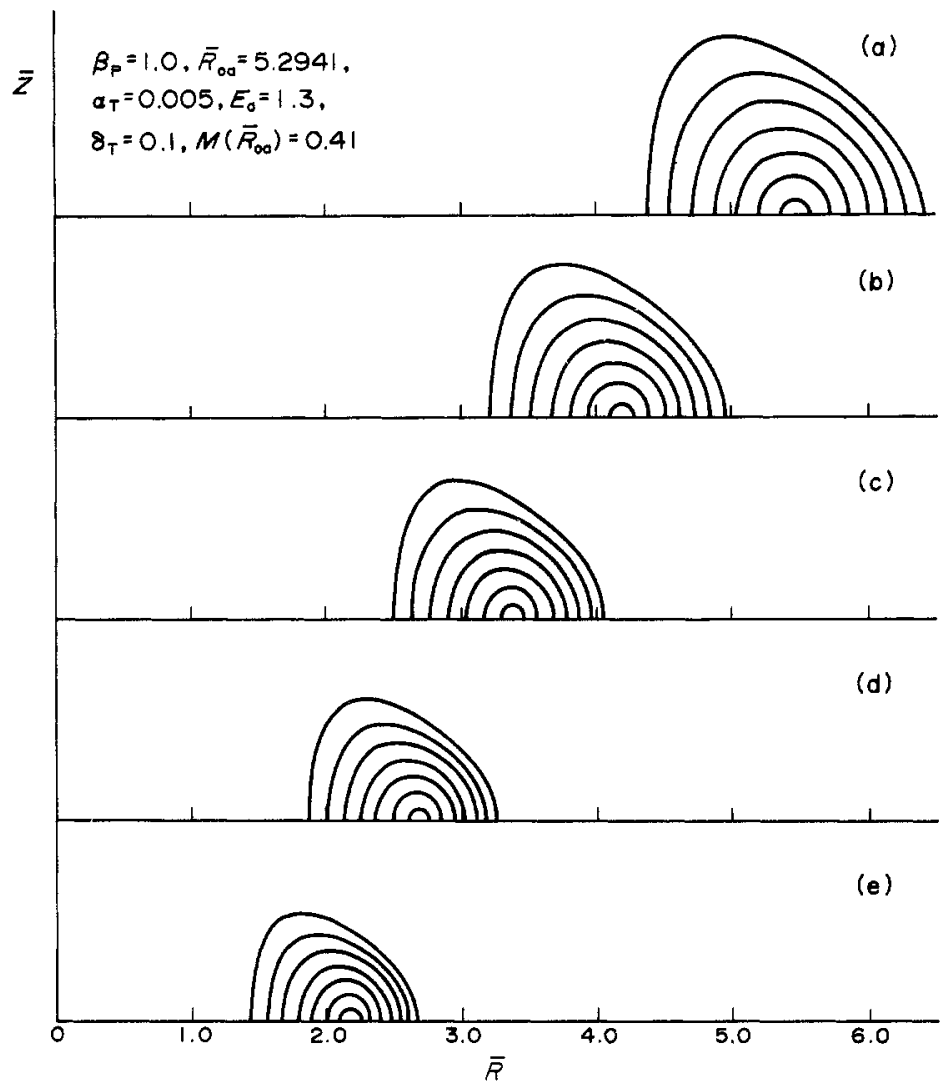

FIG. 1.--Plot of constant $\psi$ contours during compression.

Tokamaks with circular, elliptic and D-shape sections and for the adiabatic compressions of rotating plasma with small Mach number. The details are given in a separate report by one of us (D. Fu). Only the results for the adiabatic compression of rotating plasma with D-shape and Mach number 0.4 are shown in Figs 1-4 as examples. The numerical results calculated by our method and the usual two-

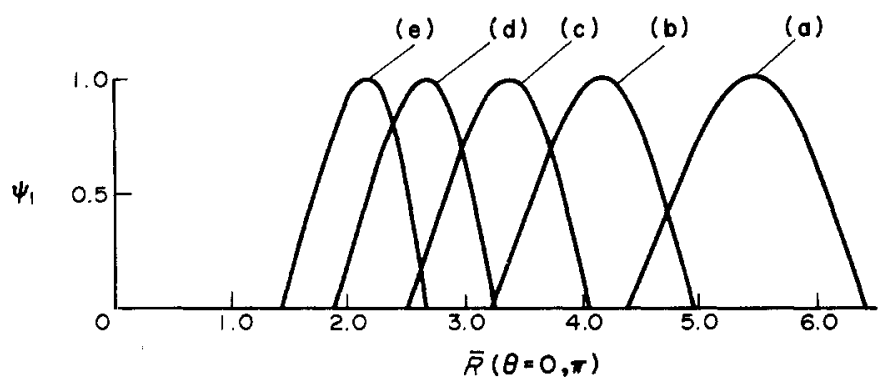

FIG. 2.-Radial distribution of magnetic flux $\psi$, at $\theta=0, \pi$. 


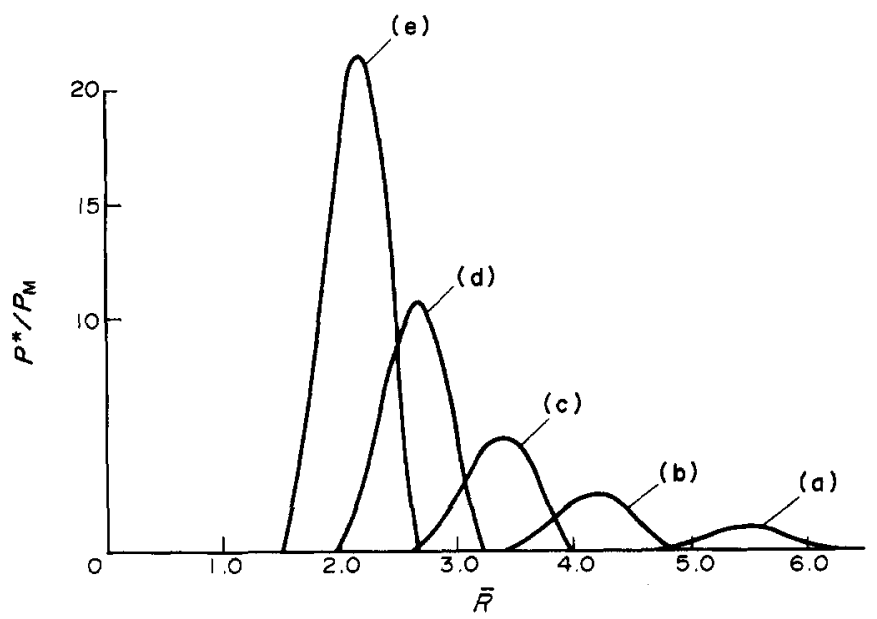

FIG. 3.-Radial distribution of the pressure of plasma at $\theta=0, \pi$.

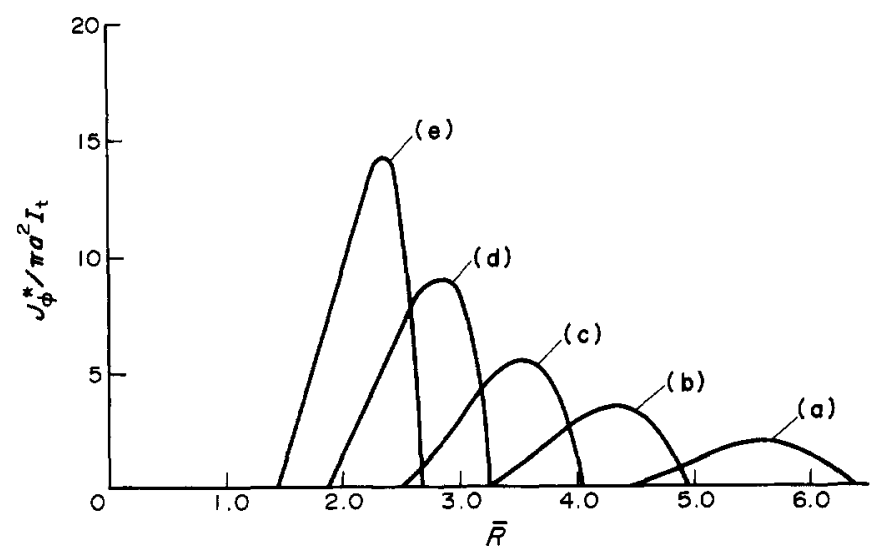

FIG. 4.-Radial distribution of the toroidal current at $\theta=0, \pi$.

dimensional method for a static equilibrium state are given in Table 2 for a comparison.

\section{CONCLUSIONS}

Table 2 shows that the numerical results calculated by our method and the usual two-dimensional method for the static equilibrium state agree well.

Figure 1 indicates the changes in $\psi$ contours during compression for the case of $\beta_{\mathrm{p}}=1, \bar{R}_{0 a}=5.294, E_{a}=1.3, \delta_{\mathrm{T}}=0.1$ and $M\left(\bar{R}_{0 a}\right)=0.41$. The final compression ratio may raise the temperature of plasma significantly.

Figures 2-4 describe the distribution of $\psi$, the pressure and the toroidal current on major radius, respectively. The pressure $p^{*}$ gets higher and higher when the plasma 
TABLE 2. - THE NUMERICAL RESULTS CALCULATED BY THE USUAL TWO-DIMENSIONAL METHOD AND THE PRESENT VARIATIONAL METHOD FOR THE STATIC EQUILIBRIUM STATE WITH $\beta_{\mathrm{p}}=1.0$, $\varepsilon=0.37307, E_{a}=1.0, \delta_{\mathrm{T}}=0$ AND $\delta^{2}=0.0025$

\begin{tabular}{lcccc}
\hline & $\beta_{\mathrm{p}}$ & $P_{\mathrm{p}}$ & $B_{\mathrm{t}}$ & $\bar{R}_{\mathrm{m}}$ \\
\hline $\begin{array}{l}\text { Two-dimensional } \\
\text { method }\end{array}$ & 0.8439 & 3.803 & 0.1875 & 2.9040 \\
$\begin{array}{l}\text { Variational method } \\
\text { Error }\end{array}$ & 0.8437 & 3.809 & 0.1821 & 2.9039 \\
& 0.0002 & 0.006 & 0.0054 & 0.0001 \\
\hline
\end{tabular}

is compressed towards the axis of symmetry, as does the current $J_{\phi}$. The rise in pressure and toroidal current result in a higher temperature.

It is found that, when the Mach number is small, for example 0.4, the effects of plasma rotation are negligible.

The present variational method may save computer time considerably.

\section{REFERENCES}

Grad H., Hu P. N. and Stevens D. C. (1975) Proc. natn Acad. Sci. U.S.A. 72, 3789

Hameiri E. (1983) Phys. Rev. A27, 1259.

Lao L. L., Hirshman S. P. and Wieland R. M. (1981) Physics Fluids 24, 1431.

Pan L. J. (1989) Plasma Phys. Contr. Fusion 31, 1005. 